\title{
Multi-Resolution Channel Codes
}

\author{
Hanying Feng ${ }^{1}$ Michelle Effros \\ Dept. of Electrical Eng. (136-93) \\ California Institute of Technology \\ Pasadena, CA91125, USA \\ email: \{fhy, effros\}@z.caltech.edu
}

\begin{abstract}
In this paper, we present a new approach for channel coding on unknown or time-varying channels. Given a family of possible channel characteristics, we define a multi-resolution channel code as a single channel coding codebook from which a collection of codes of increasing rates are extracted by choosing larger and larger nested subsets of the original set of codewords. We give an achievable rate region and a tight converse.
\end{abstract}

\section{INTRODUCTION}

Consider the problem of channel coding for an unknown or time-varying channel. Given a family of possible channel characteristics, in theory we could design a different channel code for each channel in our collection. Assuming knowledge (at both the transmitter and the receiver) of the channel in operation at communication time, both encoder and decoder could choose among the family of codes. The resulting strategy would theoretically achieve the capacity of any channel in the collection (e.g., [1], [2] and [3]). Unfortunately, this approach requires an uncountably infinite collection of channel codes when $\Omega$ is uncountably infinite. We define a multi-resolution channel code (MRCC) as a single channel coding codebook from which a collection of codes of increasing rates are extracted by choosing larger and larger nested subsets of the original set of codewords. Given a collection $\Omega$ of channels and a fixed set of $2^{n R_{\max }}$ channel codewords, the MRCC uses the first $2^{n r(\theta)}$ codewords to code at rate $r(\theta) \leq R_{\max }$ across channel $\theta \in \Omega$. We here consider the set of rate functions $r(\cdot)$ achievable on a fixed class $\Omega$ of channels. MRCCs are similar in application to punctured channel codes (e.g., [4]).

\section{Preliminaries}

Consider a class $\Omega$ of memoryless channels with common input alphabet $A$ and output alphabet $B$. For each $\theta \in \Omega$, let $C(\theta)$ and $\nu_{\theta}$ denote the capacity and conditional distribution of channel $\theta$, respectively. Given $\Omega$, a positive constant $R_{\max }$, and a rate function $r: \Omega \rightarrow\left[0, R_{\max }\right]$ that is measurable with respect to the Borel $\sigma$-algebra of open subsets on $\Omega$, a MRCC $\mathcal{C}_{n}=\left(\mathcal{F}_{n}, f_{n}, g_{n}, r\right)$ on $\Omega$ is a single channel code defined by a codebook $\mathcal{F}_{n}$, a measurable encoder $f_{n}$, and a measurable decoder $g_{n}$. The channel codebook $\mathcal{F}_{\mathfrak{n}}$ contains $\left\{2^{n R_{\max }}\right\rfloor$ blocklength- $n$ codewords. The codewords are ordered and denoted by $\mathcal{F}_{n}=\left\{a^{n}(1), \ldots, a^{n}\left(\left\lfloor 2^{n R_{\max }}\right\rfloor\right)\right\}$. The channel $\theta \in \Omega$ in operation is assumed to be fixed and known to the channel code's encoder and decoder during any single channel use. The channel may vary from channel use to channel use. For any $\theta \in \Omega$, the copde is used at rate $r(\theta)$ on channel $\theta$. The collection $U_{n}^{(\theta)}$ of allowable messages on

\footnotetext{
${ }^{1}$ This work was supported by NSF MIP-9501977 and CCR9909026 and grants from the Lee Center for Advanced Networking and the Powell Foundation.
}

$\theta$ is defined as $U_{n}^{(\theta)}=\left\{1, \ldots,\left\lfloor 2^{n r(\theta)}\right\rfloor\right\}$. For any $\theta \in \Omega$, the encoder is defined as $f_{n}(\theta, u)=a^{n}(u)$ for all $u \in U_{n}^{(\theta)}$; the corresponding decoder $g_{n}(\theta, \cdot)$ maps the channel output space $B^{n}$ back to the set $U_{n}^{(\theta)}$ of allowable messages. For any $\theta \in \Omega$ and $u \in U_{n}^{(\theta)}$, let $\Gamma_{u}^{(\theta)}=\left\{y^{n} \in B^{n}: g_{n}\left(\theta, y^{n}\right)=u\right\}$ represent the decoding cells associated with $u$ and $\theta$. Then for any class $\Omega$ of channels and MRCC $\mathcal{C}_{n}=\left(\mathcal{F}_{n}, f_{n}, g_{n}, r_{n}\right)$, we define the average probability of error of $\mathcal{C}_{n}$ on $\Omega$ with respect to $\beta$ as

$$
P_{e, \beta}^{(n)}\left(\mathcal{C}_{n}, \Omega\right)=\int_{\Omega}\left[\frac{1}{\left[2^{n r(\theta)}\right]} \sum_{u \in U_{n}^{(\theta)}} \nu_{\theta}^{n}\left(\left(\Gamma_{u}^{(\theta)}\right)^{c} \mid a^{n}(u)\right)\right] d \beta(\theta),
$$

where $\beta$ is an arbitrary distribution on $\Omega$.

A $\left(\left\lfloor 2^{n R_{\max }}\right\rfloor, n, r(\cdot), \epsilon\right)$-block $M R C C$ for $(\Omega, \beta)$ is defined as a MRCC $\mathcal{C}_{n}=\left(\mathcal{F}_{n}, f_{n}, g_{n}, r\right)$ with $P_{e, \beta}^{(n)}\left(\mathcal{C}_{n}, \Omega\right) \leq \epsilon$. For any $R_{\max }<\infty$, we call the rate function $r: \Omega \rightarrow\left[0, R_{\max }\right]$ achievable on $\Omega$ if for any distribution $\beta$ there exists a sequence of $\left(\left\lfloor 2^{n R_{\max }}\right\rfloor, n, r_{n}(\cdot), \epsilon_{n}\right)$-block MRCCs with respect to $\beta$ on $\Omega$ such that $\lim _{n \rightarrow \infty} r_{n}(\theta)=r(\theta)$ for each $\theta \in \Omega$ and $\lim _{n \rightarrow \infty} \epsilon_{n}=0$.

\section{RESULTS}

Theorem 1 If $\Omega$ is a collection of stationary, memoryless channels such that $\max _{\theta \in \Omega} C(\theta)<\infty$, then

$$
r(\theta)=C(\theta) \quad \forall \theta \in \Omega
$$

is achievable if one of the following holds: (1) $\Omega$ is finite; (2) the channel input alphabet is finite; (3) the optimal input distribution for each $\theta \in \Omega$, has a bounded derivative and power.

Theorem 2 If $\Omega$ is a collection of stationary, memoryless channels such that $\max _{\theta \in \Omega} C(\theta)<\infty$ and the power budget is $P(\theta)$ for any $\theta \in \Omega$, then

$$
r(\theta)=C(\theta) \quad \forall \theta \in \Omega
$$

is achievable if one of the following holds: (1) $\Omega$ is finite; (2) the channel input alphabet is finite; (3) for each $\theta \in \Omega, \mu_{\theta}(x)$, the optimal input distribution for channel $\theta$ has a bounded derivative and for any $\epsilon>0$, there exists an $S_{a}$ such that $\int_{|x|>S_{a}} x^{2} \mu_{\theta}(x) d x<\epsilon$

\section{REFERENCES}

[1] A. J. Goldsmith and M. Médard, Capacity of Time-Varying Channels with Causal Channel Side Information, Preprint.

[2] G. Caire and S. Shamai, On the capacity of some channels with channel state information, IEEE Trans. Inform. Theory, vol. 45, pp. 2007-2019, Sep., 1999.

[3] J. Wolfowitz, Coding Theorems of Information Theory, Springer-Verlag, 3rd edition, 1978.

[4] S. B. Wicker, Error Control Systems for Digital Communication and storage, Prentice-Hall, Englewood Cliffs, NJ, 1995. 